\title{
Robust Carbon Nitride-Based Thermoset Coatings for Surface Modification and Photochemistry
}

\author{
Baris Kumru, ${ }^{\dagger}$ Jesús Barrio, ${ }^{\ddagger}$ Jianrui Zhang, ${ }^{\dagger}$ Markus Antonietti, ${ }^{\dagger}$ Menny Shalom, ${ }^{*},+\odot$ \\ and Bernhard V. K. J. Schmidt* ${ }^{*} \dagger$ \\ ${ }^{\dagger}$ Max Planck Institute of Colloids and Interfaces, 14424 Potsdam, Germany \\ ${ }^{\ddagger}$ Department of Chemistry and Ilse Katz Institute for Nanoscale Science and Technology, Ben-Gurion University of the Negev, \\ 8410501 Beer-Sheva, Israel
}

Supporting Information

ABSTRACT: Herein, the convenient visible light-induced photografting of hydroxyl ethyl methacrylate onto graphitic carbon nitride ( $\mathrm{g}-\mathrm{CN})$ is described, leading to well-dispersible $\mathrm{g}$-CN-based precursor polymers that can be injected. Mixing with citric acid as the cross-linker and heating leads to stable thermoset coatings. The process is versatile and easy to perform, leading to g-CN-based coatings. Moreover, the coating can be further functionalized/modified via grafting of other polymer chains, and the resulting structure is useful as photocatalytic

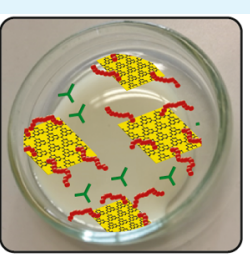

$\mathrm{CN}$-precursor

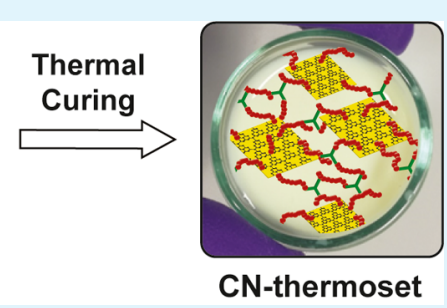
surface or as photoelectrode.

KEYWORDS: carbon nitride, dispersion, coating, thermoset, photoactive surface, photoelectrochemistry, photopolymerization

\section{INTRODUCTION}

Photoactive nanomaterials have been in the focus of nanoscience in recent years. ${ }^{1,2}$ Among the various photoactive materials, quantum dots, ${ }^{3,4}$ conjugated polymers, ${ }^{5,6}$ or metal oxides $^{7,8}$ have been studied very frequently. In particular, applications such as bioimaging, ${ }^{9,10}$ energy conversion, ${ }^{11}$ or photocatalysis $^{12}$ are intriguing and drive the research forward. A frequently investigated material is graphitic carbon nitride (g-CN), which is mainly because of its photocatalytic and chemical properties as well as facile synthesis. ${ }^{13-16} \mathrm{~g}-\mathrm{CN}$ has been utilized as the catalyst in applications such as $\mathrm{CO}_{2}$ conversion, ${ }^{17,18}$ hydrogen evolution, ${ }^{19,20}$ synthesis of organic molecules, ${ }^{21,22}$ or as promoter for the photoinitiation of polymerizations. ${ }^{23-25}$ Recently, porosity and grain size of $\mathrm{g}$ $\mathrm{CN}$ was correlated with hydrogen evolution efficiency, which shows how the material textures affect the utility of $\mathrm{g}-\mathrm{CN}{ }^{26}$ Moreover, g-CN was doped with metals to gain access to diversified catalysis mechanisms in antibiotic degradation. ${ }^{27}$ In electro-oxidation of formic acid or methanol, g-CN was combined with Pd and carbon black to obtain stable and reliable catalysts. ${ }^{28}$ One of the major disadvantages of g-CN lies in its low dispersibility in water or organic solvents. The latter limits its maximum concentration and the range of applications. Thus, various approaches have been investigated to tackle the dispersibility issue, for example, surface functionalization $^{29-31}$ or treatment with strong acids, ${ }^{32}$ just to name a few. Recently, photo-induced functionalization reaction has been introduced as a versatile tool to enhance dispersibility of g-CN and tailor the surface structure according to specific needs. ${ }^{23,33-35}$ In addition, photoreactive surfaces have found significant interest recently. ${ }^{36,37}$ In such a way, surface properties can be altered effectively and with spatial control, for example, for polymer grafting, ${ }^{38-40}$ placement of cells, ${ }^{41,42}$ protein functionalization, ${ }^{43}$ or light-emitting diodes (LEDs). ${ }^{44}$

g-CN has remarkable photocatalytic properties; ${ }^{45}$ thus, the formation of g-CN films and coatings is a topic of significant interest for further exploitation of $\mathrm{g}-\mathrm{CN}$ in photoelectric devices. An early example of g-CN film formation utilized a sputtering approach that allowed the formation of uniform coatings with thicknesses up to $2 \mu \mathrm{m}$. ${ }^{46}$ One of the methods that are frequently used is based on vapor deposition, which allows film formation on various substrates such as indium tin oxide, silica, or glass. ${ }^{47,48}$ Such g-CN films can be utilized as actuators reacting to various external triggers. ${ }^{47}$ Wang and coworkers investigated the formation of g-CN films and coatings via the formation of a sol. ${ }^{49}$ In this work, the sol was formed via oxidation of the g-CN in an acidic environment. Another approach is the direct growth of $\mathrm{CN}$ on the surface, for example, via a supramolecular preorganization route or precursor paste formation. ${ }^{50-52}$ Wang and co-workers showed the catalytic activity of g-CN films in water splitting. ${ }^{53}$ The films were formed on fluorine-doped tin oxide glass directly, which formed an effective system for photocatalysis. Structured and patterned surfaces were generated via templating methods, for example, soft or hard templating as well as a combination of both, ${ }^{54-56}$ an additional way to patterned surface growth of g$\mathrm{CN}$ inside well-defined porous substrates. ${ }^{57}$ Moreover, film

Received: December 11, 2018

Accepted: February 12, 2019

Published: February 12, 2019 
Scheme 1. Schematic Overview of CM-HEMA Precursor Formation and Subsequent Film Formation via Thermal Curing
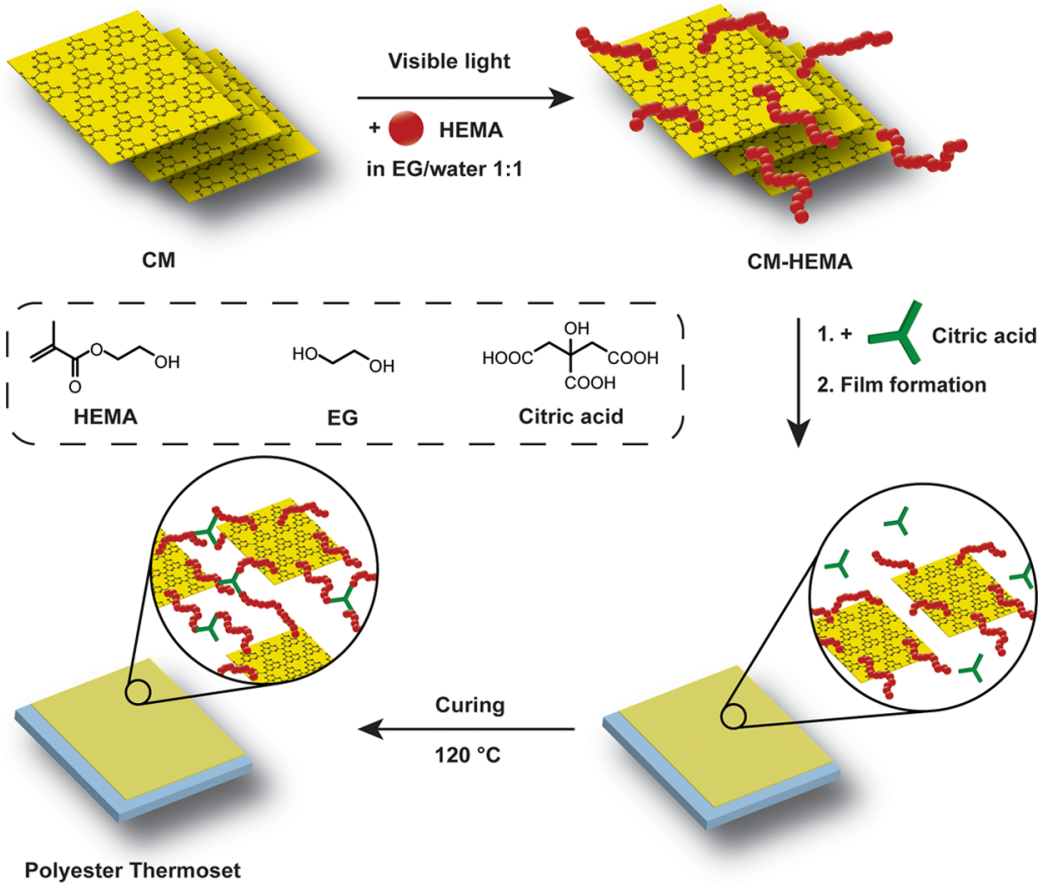

formation enables the formation of flexible devices if flexible substrates are employed, which is of significant interest for organic electronics. Nevertheless, the formation of g-CN containing polymer coatings and films in a straightforward and easy way is still a matter of research. Hence, film formation can be consigned to a polymer, whereas the photochemical and catalytic properties are provided by the g-CN. Here, we approach these coatings by embedding g-CN into a polyester thermoset, which is a fundamentally different approach compared to literature-known g-CN films. The polymerbased route is largely scalable, reproducible, and affordable. Moreover, the utilization of a polymer matrix allows the formation of various shapes and structures, which is hardly achieved with g-CN itself. Polyesters are common polymer materials that have various applications in everyday life, and they are considered to be robust against $\mathrm{CN}$ oxidation. Using colloidal precursors, carbon materials were introduced into polyester thermosets for enhancing mechanical properties, ${ }^{58}$ or polyester thermosets were formed from renewable resources. ${ }^{59}$ Polyester thermosets combined with inorganic compounds can be indeed considered model systems to obtain hybrid materials with enhanced mechanical and thermal properties. ${ }^{60,61}$

Herein, the convenient photografting of hydroxyl ethyl methacrylate (HEMA) onto g-CN is described, leading to welldispersible g-CN precursor colloids [cyanuric acid-melamine (CM)-HEMA] (Scheme 1) that can be processed or injected. The precursors can be mixed with citric acid as a cross-linker and converted into stable coatings via heating. Moreover, the coating can be further functionalized/modified via grafting of other polymer chains, utilizing the photocatalytic surface.

\section{EXPERIMENTAL SECTION}

Materials. $\mathrm{Al}_{2} \mathrm{O}_{3}$ basic (Sigma-Aldrich), citric acid (ACS grade 99.5\%, Sigma-Aldrich), cyanuric acid (98\%, Sigma-Aldrich), $\mathrm{D}_{2} \mathrm{O}$ (99.9\%, Sigma-Aldrich), dichloromethane (DCM, anhydrous HPLC grade, Merck), ethylene glycol (EG, 99\%, Fluka), hydrochloric acid (1 $\mathrm{M}$ solution, Merck), hydroquinone (ReagentPlus, Sigma-Aldrich), melamine (99\%, Sigma-Aldrich), potassium hydroxide (SigmaAldrich), rhodamine $\mathrm{B}(\mathrm{RhB},>95 \%$ Sigma-Aldrich), and tetrahydrofuran (THF, anhydrous, Sigma-Aldrich) were used as purchased. 2-HEMA (99\%, Sigma-Aldrich), N,N-dimethylacrylamide (DMA, 99\%, Sigma-Aldrich), and styrene (ReagentPlus, Sigma-Aldrich) were passed through basic alumina column prior to use to remove the inhibitor. For visible light irradiation, $50 \mathrm{~W}$ LED chips (Foxpic High Power 50 W LED Chip Bulb Light DIY White 3800LM $6500 \mathrm{~K}$ ) were connected to a self-made circuit and cooling system. $\mathrm{g}-\mathrm{CN}$ from the cyanuric acid-melamine complex (noted as CM) was synthesized according to the literature. ${ }^{62}$

Synthesis of CM-Based Precursor. CM (200 mg) was mixed with $4.5 \mathrm{~g}$ of deionized water and $4.5 \mathrm{~g}$ of EG and ultrasonicated at $50 \%$ amplitude for $20 \mathrm{~min}(10 \times 2 \mathrm{~min}$ portions $)$ to yield a g-CN dispersion. Afterward, $0.8 \mathrm{~g}$ of HEMA was added to the dispersion, and the mixture was put between two $50 \mathrm{~W}$ LED daylight sources $(20$ $\mathrm{cm}$ apart from each other) and reacted for $3 \mathrm{~h}$ under mild stirring (conversion $94 \%$ obtained via ${ }^{1} \mathrm{H}$ NMR). The resulting highly viscous and disperse material was later utilized as the coating precursor after the addition of citric acid.

Synthesis of $\mathrm{g}$-CN Coating. The g-CN-based precursor $(2 \mathrm{~g})$ was mixed with $100 \mathrm{mg}$ of citric acid and stirred until dissolved. Later on, the precursor mixture can be applied to the desired surface and cured at $120{ }^{\circ} \mathrm{C}$ for $3 \mathrm{~h}$ for complete cross-linking and evaporation of solvents.

Further Polymer Grafting on CM-HEMA Coatings. CM-HEMAcoated glass substrate surfaces were covered with monomer (DMA or styrene) and illuminated via visible light for $8 \mathrm{~h}$ from the top (illumination applied from $20 \mathrm{~cm}$ above the sample). After reaction, the DMA-grafted substrate was washed with $20 \mathrm{~mL}$ of distilled water, whereas the styrene-grafted substrate was washed with $20 \mathrm{~mL}$ of THF for the removal of unreacted/nongrafted impurities.

Dye Degradation via CM-HEMA Coatings. The photoactivity of the CM-HEMA films was tested by the adsorption of an organic dye and its degradation under white light irradiation. For this experiment, glass-coated CM-HEMA films $\left(2 \times 5 \mathrm{~cm}^{2}\right)$ were submerged in a solution of $\mathrm{RhB}\left(20 \mathrm{mg} \mathrm{mL} \mathrm{m}^{-1}\right)$ overnight and then exposed to illumination with a $50 \mathrm{~W}$ white LED array (Bridgelux BXRA-50C300; $\lambda>410 \mathrm{~nm}$ ). The degradation of the dye to $\mathrm{CO}_{2}$ with the CMHEMA films was confirmed by gas chromatography (GC). 

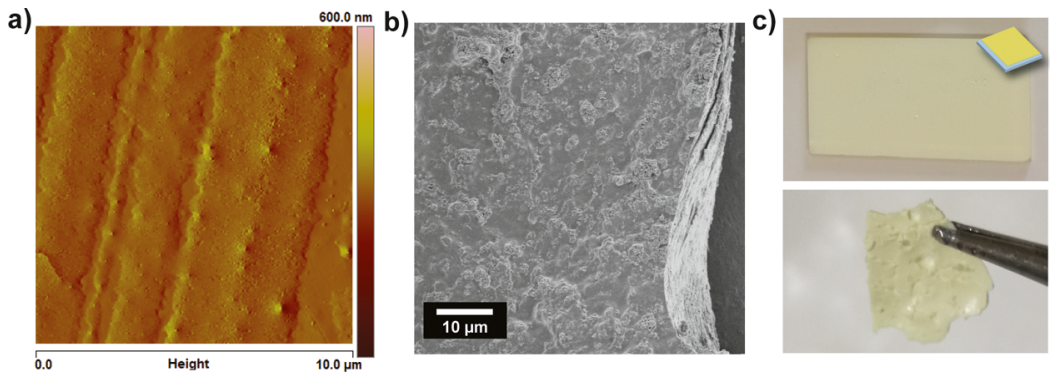

Figure 1. Characterization of CM-HEMA films: (a) AFM imaging, (b) SEM imaging, and (c) photographs of glass-coated substrate and a freestanding film.

Photoelectrochemistry. Photoelectrochemical measurements were recorded using a three-electrode system on an Autolab potentiostat (Metrohm, PGSTAT 101). A Pt foil electrode and an $\mathrm{Ag} / \mathrm{AgCl}$ (3 M $\mathrm{KCl})$ electrode were used as the counter and reference electrode, respectively. Photocurrent measurements were obtained in $0.5 \mathrm{M}$ $\mathrm{H}_{2} \mathrm{SO}_{4}, \mathrm{pH} 0.2$ aqueous solution under one sun illumination (1.5 AM). The electrode was prepared by depositing the CM-HEMA precursor mixture on a fluorine-doped tin oxide (FTO) electrode. The prepared CM-HEMA/citric acid coating precursor $(1 \mathrm{~mL})$ was drop-casted over the conductive side of the FTO electrode and heated at $100{ }^{\circ} \mathrm{C}$ with a heating plate until thin, homogeneous films were obtained.

Characterization Methods. Scanning electron microscopy (SEM) was performed using JSM-7500F (JEOL) equipped with an Oxford Instruments X-MAX $80 \mathrm{~mm}^{2}$ detector for g-CN-based coating surface morphology. Fourier transform infrared (FT-IR) spectra were taken on Nicolet iS 5 FT-IR spectrometer. Sessile drop contact angles of water on the coatings were measured at room temperature about 5-10 s after placing the drop on the surface with a DSA 10 video contact angle measuring system G10 (Krüss, Germany), and data evaluation was done with software DSA version 1.80.02. Thermogravimetric analysis (TGA) was performed via TG 209 Libra from Netzsch in a nitrogen atmosphere with a heating rate of $10 \mathrm{~K} \mathrm{~min}^{-1}$ using an aluminum crucible for samples. Ultrasonication was performed via an ultrasonicator at 50\% amplitude (Branson D450). For rheological investigations, Anton Paar MCR 301 rheometer equipped with a cone plate $12(\mathrm{CP}-12)$ was used. Measurements were performed at a constant angular frequency $\left(10 \mathrm{rad} \mathrm{s}^{-1}\right)$ with a strain range from 0.1 to $100 \%$ with 31 measuring points and $0.02 \mathrm{~mm}$ gap. Frequency-dependent measurements were performed at constant strain $(0.1 \%)$ with changing frequency in the range of $1-100 \mathrm{rad} \mathrm{s}^{-1}$. Viscosity measurements were performed at ambient temperature with changing shear rates between 1 and $40 \mathrm{~s}^{-1}$. The flow curve was obtained via measuring the shear stress and viscosity with changing shear rates from 0 to $100 \mathrm{~s}^{-1}$. Atomic force microscopy (AFM) from Digital Instruments was employed for the investigation of half-coated glass samples. GC was performed with an Agilent 7820 GC System. Elemental analysis was performed with a Vario microdevice. The sample for elemental analysis was prepared from an elastic CMHEMA film (without citric acid cross-linking) after dissolution in methanol, precipitation in THF, and drying under vacuum. ${ }^{1} \mathrm{H}$ NMR at $400 \mathrm{MHz}$ on an Ascend 400 from Bruker was utilized for determination of HEMA conversion in $\mathrm{D}_{2} \mathrm{O}$.

\section{RESULTS AND DISCUSSION}

As a starting material, g-CN was synthesized from the cyanuric acid-melamine complex via heat treatment. The final $\mathrm{g}-\mathrm{CN}$ product will be annotated as CM for the rest of the article. ${ }^{62}$ The synthesized CM presents the typical absorption and X-ray diffraction (XRD) profile, that is, an absorption band between 280 and $400 \mathrm{~nm}$ and XRD peaks at $13^{\circ}$ (intraplanar heterocyclic stacking) and $28^{\circ}$ (interplanar stacking) (Figure S1). To form CM-based coatings, $\mathrm{CM}$ was grafted with HEMA - a hydroxyl containing monomer-via a free radical polymerization pathway initiated with visible light. To facilitate optimum conditions, a 1:1 mixture of water and EG was utilized as the reaction medium that allows to increase the solid content of the reaction via enhanced dispersibility of $\mathrm{CM}^{35}$ Hence, HEMA-grafted CM (CM-HEMA) could be obtained in a facile fashion. To characterize the formed material and prove successful grafting, FT-IR and elemental analysis were performed (Figure S2 and Table S1). FT-IR showed the appearance of bands that can be assigned to poly(HEMA) (PHEMA), for example, the band around 1750 $\mathrm{cm}^{-1}$ corresponding to the carbonyl stretching vibration (Figure S2). Both CM and CM-HEMA materials present absorption bands corresponding to the triazine breathing of $\mathrm{CN}$ around $800 \mathrm{~cm}^{-1}$. Moreover, stretching modes of $\mathrm{CN}$ heterocycles can be detected between 1150 and $1600 \mathrm{~cm}^{-1}$, and asymmetric $\mathrm{CH}$ stretching arising from HEMA can be observed around $2900 \mathrm{~cm}^{-1}$ in CM-HEMA (Figure S2). XRD of the CM-HEMA precursor shows no crystallinity probably because of the undefined nature of the material before crosslinking (Figure S3). X-ray photoelectron spectroscopy proves the formation of modified g-CN films (Figure S4). C 1s spectra display three different chemical states corresponding to $\mathrm{sp}_{3} \mathrm{C}-$ $\mathrm{C}(284.5 \mathrm{eV})$ and $\mathrm{C}-\mathrm{O}(286.0 \mathrm{eV})$ bonds, which confirm the presence of high amounts of the HEMA precursor. Furthermore, the contribution at higher binding energies $(288.7 \mathrm{eV})$ certifies the presence of $\mathrm{C}=\mathrm{N}-\mathrm{C}$ units within the films. $\mathrm{N} 1 \mathrm{~s}$ spectra show the typical features of $\mathrm{CN}$ materials; ${ }^{63,64}$ three different contributions can be observed that belong to $\mathrm{C}=\mathrm{N}-\mathrm{C}$ coordination $(398.7 \mathrm{eV}), \mathrm{N}-(\mathrm{C})_{3}$ $(399.9 \mathrm{eV})$, and the remaining amino groups $(\mathrm{N}-\mathrm{H}, 401.0$ $\mathrm{eV})$.

To gain insights into the polymerization process and to verify the free radial polymerization mechanism, blank reactions were performed. Without light irradiation, no conversion of monomer was observed (Figure S5). After the addition of a polymerization inhibitor, namely hydroquinone, insignificant conversions (below 5\%) compared to the inhibitor-free polymerization (conversion above 90\%) were observed. The dispersion of CM-HEMA in water/EG medium could be utilized directly as precursor for coatings. Citric acid was added as a cross-linker to obtain stable coatings. An efficient coating process was established via adjustment of the polymerization conditions to yield CM-HEMA precursor with a suitable viscosity for film formation (Figure S6) that features values around $25 \mathrm{~Pa} \mathrm{~s}$ at a shear rate of $1 \mathrm{~s}^{-1}$ and a storage modulus $\left(G^{\prime}\right)$ of $5550 \mathrm{~Pa}$ at $1 \%$ strain (Figures $\mathrm{S} 7$ and S8). As the $G^{\prime}$ is higher than the loss modulus $\left(G^{\prime \prime}\right)$, the precursor has viscoelastic behavior. Significant shear thinning was observed leading to $G^{\prime}$ values of $68 \mathrm{~Pa}$ at $50 \%$ strain. Between 32 and $40 \%$ strain, the gel-like behavior shifts to a sol- 


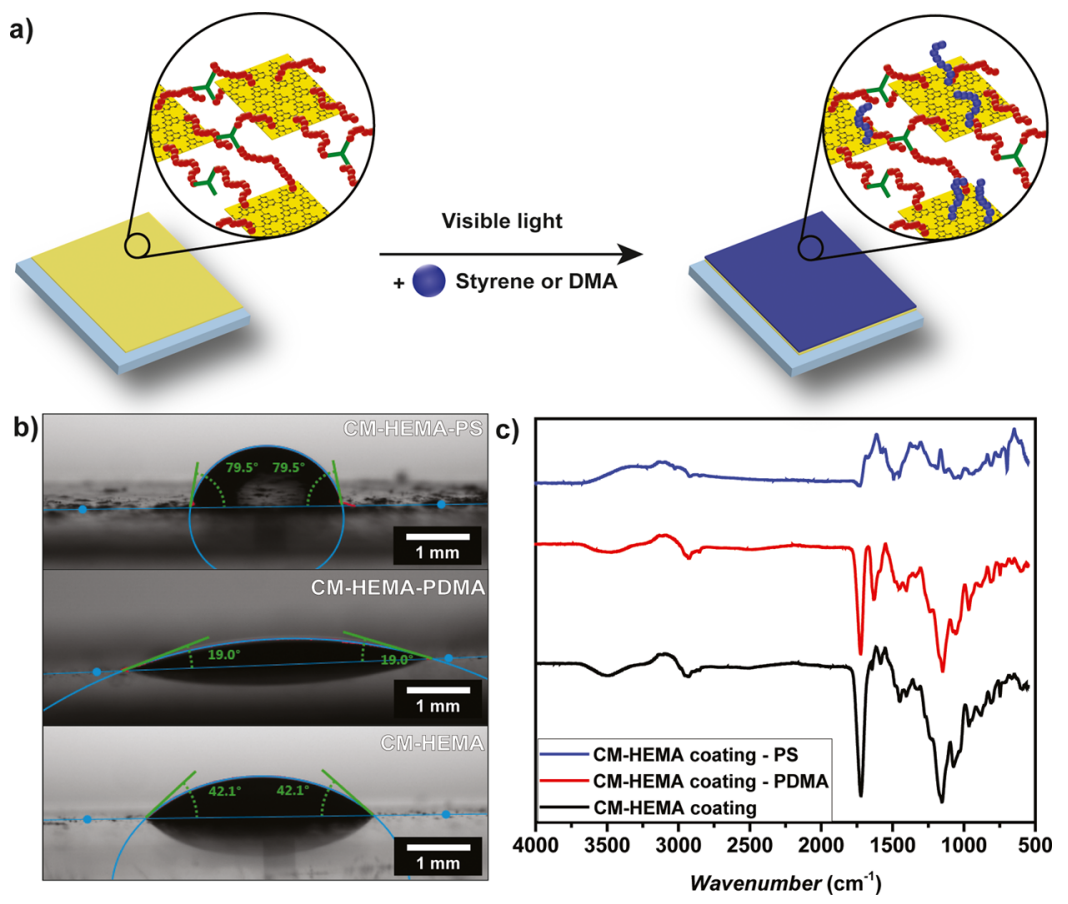

Figure 2. Coating of CM-HEMA films with PS and PDMA: (a) schematic overview of the grafting process, (b) contact angle measurements (bottom: CM-HEMA; middle: CM-HEMA-PDMA; top: CM-HEMA-PS), and (c) FT-IR results.

like behavior $\left(G^{\prime \prime}>G^{\prime}\right)$ (Figure S8). The precursor was obtained as a yellow viscous dispersion that can be processed and even injected with a syringe as well (Figure S6), utilizing the shear-dependent viscosity of the precursor (Figure S7), for example, at a shear rate of $15 \mathrm{~s}^{-1}$, the viscosity drops to $1.3 \mathrm{~Pa}$ s. This way, the precursor can be applied to various surfaces in a spatially controlled manner. Viscosity values at a low shear range is needed for applications via dipping, however, significantly decreased viscosity at higher shear rates provides plausible applications via spraying or injection.

To facilitate the cross-linking, a carboxylic acid cross-linker such as citric acid was added to the CM-HEMA dispersion. After its formation, the film could be cured via thermal treatment at $120{ }^{\circ} \mathrm{C}$ to form the cross-linked polyester (Scheme S1). Homogenous films were obtained after curing as observed via AFM and SEM (Figure 1). AFM shows a smooth film with homogenous thicknesses around $500 \mathrm{~nm}$ when compared to the uncoated glass slide (Figure S9). The AFM results are confirmed by SEM that shows a film with minor surface alteration and nonporous as well as rigid structure (Figures 1 and S10). The thickness of films can be adjusted conveniently via the applied method (spraying or brushing) and the applied cycles. However, a detailed study regarding the thickness and photocatalytic properties is work in progress. The incorporation of CM was observed via FT-IR as well as the naked eye because of the light-yellow color of the films. $\mathrm{XRD}$ of the coating is dominated by the amorphous polymeric network and shows only a minor diffraction peak at $28^{\circ}$ (Figure S11). Interestingly, films could be formed and cured on various substrates, for example, on glass, copper, poly(styrene) (PS), or wood, which shows the versatility of the presented method (Figures S12 and S13). In addition, the formed coatings can be detached from the substrate, leading to a free-standing film (Figure 1). The cross-linking process can be followed easily via TGA. A weight loss of around $87 \%$ can be observed between 50 and $170{ }^{\circ} \mathrm{C}$ with the steepest weight loss around $139{ }^{\circ} \mathrm{C}$ (Figures S14 and S15). There are several processes happening during heating of the precursor mixture. First of all, solvent, that is, water and EG, evaporates. Second, a condensation reaction between citric acid, CM-HEMA, and most likely EG takes place. The final product consists of a polyester between citric acid, EG, and PHEMA. To quantify the incorporation of EG, gravimetry was utilized. After curing at $120{ }^{\circ} \mathrm{C}$ for $24 \mathrm{~h}$, an EG incorporation of $52 \mathrm{wt} \%$ was observed (refer to the Supporting Information for details of the calculation). Thus, EG acts as a bridging molecule to obtain an extended cross-linked gel-like structure that enables the photochemical features of the coating (see below). The curing temperature of $120^{\circ} \mathrm{C}$ is an intermediate temperature below the temperature of significant evaporation of solvent molecules that allows slow curing to facilitate homogenous coatings. The rather low-curing temperature for the polyester formation can be explained with the organocatalytic property of $\mathrm{CN}$ in esterification. ${ }^{65,66}$ In the absence of citric acid, curing yields a flexible film that is soluble in water (Figure S16). As soluble structures are less applicable as coatings, further investigations were focused on cross-linked structures.

The final coating has a thermal stability up to temperatures of around $290{ }^{\circ} \mathrm{C}$ (Figure S11), which is sufficient for many applications, especially in photocatalysis, where usually the temperature ranges from ambient to $120{ }^{\circ} \mathrm{C}$. The stability of the CM-HEMA coatings against chemical exposure was investigated as well. For that, films on glass slides were introduced into various chemical environments, namely water, acid ( $1 \mathrm{M} \mathrm{HCl})$, base (2 M KOH), THF, and DCM. Excluding the base treatment, in all cases, no response of the films was observed (Figure S17) also after treatment with visible light (Figure S18). Treatment with a base led to the complete dissolution of the films after $24 \mathrm{~h}$, which is due to the esterbased linkage of the coatings. As esters can be readily hydrolyzed via basic saponification, the low stability in basic medium was expected. ${ }^{67}$ In comparison, a reference film from 
linear PHEMA dissolves in organic solvents easily, which clearly supports the colloidal cross-linking also by the $\mathrm{CN}$ based precursor (Figure S19).

To study whether the formed CM-HEMA films are still photoactive, photopolymerization was probed. Therefore, CM-HEMA films were swollen with a hydrophobic monomer, styrene, and irradiated with visible light. A photoinitiated growth of PS from the coated layer could be observed via various methods. For example, contact-angle measurements show an increase in water contact angle from $42.1^{\circ}$ to $79.5^{\circ}$ (Figure 2b), which is in accordance with an increase in hydrophobicity of the surface. Moreover, the grafted PS could be located via FT-IR showing both bands from the CMHEMA precursor as well as PS (Figure $2 b$ ), for example, the HEMA carbonyl stretching band around $1750 \mathrm{~cm}^{-1}$ and the aromatic stretching of PS around $1500 \mathrm{~cm}^{-1}$. Notably, the coating could be functionalized with poly $(N, N$-dimethylacrylamide) (PDMA) via visible light photopolymerization as well. The hydrophilic PDMA leads to a decrease in water contact angle to $19.0^{\circ}$ as expected because of its hydrophilic nature (Figure 2b). FT-IR revealed the incorporation of PDMA, for example, both carbonyl stretching band from PDMA and HEMA are visible around 1650 and $1750 \mathrm{~cm}^{-1}$, respectively. Because of the photo-induced process of polymer grafting, patterned surfaces could be prepared as well. For example, a glass slide was coated with CM-HEMA, cured, and one-half was covered. Subsequently, PS was grafted in the uncovered area, followed by covering of the PS-grafted area, and another step of PDMA grafting was performed. In such a way, a surface containing a domain of PS and a domain of PDMA were prepared that have distinct different water contact behavior in two domains (Figure S20).

Functionalization of CM-HEMA films with PS and PDMA does not only prove the photoactivity of the incorporated CM but allows for versatile modification of the coating properties. In such a way, surface properties and selectivity can be tailored according to an envisioned application and combined with a photocatalytic activity in a convenient way.

The incorporated CM does not only act as a photoinitiator but can be utilized for photocatalysis as well. To test the photoactivity of the as-prepared films, we performed the uptake followed by the photodegradation of $\mathrm{RhB}$ dye. For that, a CM-HEMA-coated glass slide was immersed overnight in an $\mathrm{RhB}$ solution to achieve a complete adsorption of dye within the films. After the uptake, the films obtain the typical pink color of the organic dye (Figure 3), and the photochemical experiment starts by switching on a white LED. The film showed an excellent photocatalytic activity, degrading the dye to $\mathrm{CO}_{2}$ in $90 \mathrm{~min}$, as confirmed by the analysis of the head space during illumination by GC. Clearly, the color of the dye vanished, and the CM-HEMA film recovered its pristine yellowish color. Furthermore, the coated glass slide can be recycled and utilized in consecutive cycles of dye adsorption and photodegradation maintaining the initial activity. On the downside, the photocatalytic activity decreases compared to pure g-CN films. Nevertheless, this experiment shows one of the key futures of these CM-containing coatings: they are easily applied onto various surfaces and can act as a recyclable photocatalyst, which might be interesting for waste water remediation in the future.

In addition to photocatalysis, $\mathrm{CN}$ photoelectrodes have attracted widespread attention lately, and achieving uniform $\mathrm{CN}$ layers is one of the main challenges to address in $\mathrm{CN}$ - a)
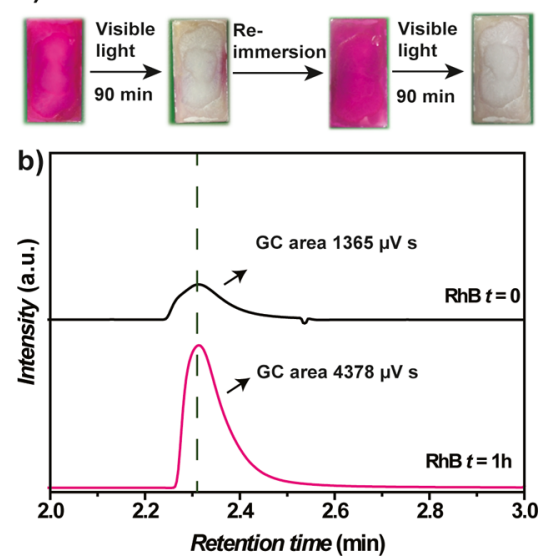

Figure 3. Photocatalytic activity of CM-HEMA films: repeated dye degradation of $\mathrm{RhB}$ (a) and GC traces of the headspace (b).

based photoelectrochemical cells. ${ }^{68,69}$ Therefore, photoelectrochemical measurements were performed, employing our coating as photoelectrode. To obtain a photoelectrode, CMHEMA precursor was deposited on an FTO electrode and thermally cured. The photoresponse was evaluated in a photoelectrochemical cell with an $\mathrm{Ag} / \mathrm{AgCl}$ reference electrode and a $\mathrm{Pt}$ counter electrode employing an acidic electrolyte $(0.5$ $\mathrm{M} \mathrm{H}_{2} \mathrm{SO}_{4}, \mathrm{pH}=0.2$ ) and one sun illumination (1.5 A) (Figure S21). Stable photocurrent was observed with values in the range of $0.3-0.7 \mu \mathrm{A} \mathrm{cm} \mathrm{cm}^{-2}$ (Figure S22). The value of the observed photocurrent strongly depends on the thickness of the layer as observed via a comparison of illumination from the front or the back. In the case of back illumination, the light has a shorter path through the photocatalytic material, which leads to an approximately doubled photocurrent. The observed photocurrents are significantly lower than those reported in literature. $^{70,71}$ A reason for the low photocurrents might be the low conductivity of the synthesized CM-HEMA coatings, which hinders effective charge transport. Although the obtained photocurrent does not reach literature values, the results are still relevant, considering the otherwise electrically insulating character of the polyester film matrix. An option for the future improvement of photoelectrochemically active layers might be the incorporation of conductive additives or more conductive polymers. As porosity is an important factor for photocatalytic activity, controlling the cross-linking density or utilization of porogens during curing might be an option to enhance the performance of g-CN-based polyester materials in the future.

\section{CONCLUSIONS}

In conclusion, a convenient method to obtain $\mathrm{CN}$-containing polymer coatings was presented. The CM-HEMA precursor can be synthesized easily via visible light irradiation, processed, injected, or printed, for example, for spatially controlled deposition. Curing is performed via heat treatment leading to smooth and stable coatings. Furthermore, the coatings can be functionalized with polymers via visible light-induced freeradical polymerization, as shown with PS and PDMA. The coatings slightly swell and are thereby are not only active in polymerization reactions but also in photocatalysis, as shown via dye degradation experiments and photoelectrochemical measurements. Overall, the CM-HEMA can be utilized to 
cover surfaces on a large scale in a convenient way and serve as a kind of platform to obtain photoactive coatings.

\section{ASSOCIATED CONTENT}

\section{S Supporting Information}

The Supporting Information is available free of charge on the ACS Publications website at DOI: 10.1021/acsami.8b21670.

Additional analytical data (rheology, viscosimetry, photographs, and photoelectrochemistry) and procedures (PDF)

\section{AUTHOR INFORMATION}

\section{Corresponding Authors}

*E-mail: mennysh@bgu.ac.il (M.S.).

*E-mail: bernhard.schmidt@mpikg.mpg.de (B.V.K.J.S.).

\section{ORCID}

Jesús Barrio: 0000-0002-4147-2667

Markus Antonietti: 0000-0002-8395-7558

Menny Shalom: 0000-0002-4506-4177

Bernhard V. K. J. Schmidt: 0000-0002-3580-7053

\section{Author Contributions}

B.K. and J.B. contributed equally. The manuscript was written through contributions of all authors. All authors have given approval to the final version of the manuscript.

\section{Notes}

The authors declare no competing financial interest.

\section{ACKNOWLEDGMENTS}

B.K. and B.V.K.J.S. acknowledge the Max-Planck Society for funding. M.S. and J.B. thank the support of the Israel Science Foundation (ISF), grant no. 1161/17, and the Minerva Center no. 117873. Open Access funding provided by the Max-Planck Society.

\section{REFERENCES}

(1) Lin, Y.; Ren, J.; Qu, X. Catalytically Active Nanomaterials: A Promising Candidate for Artificial Enzymes. Acc. Chem. Res. 2014, 47, 1097-1105.

(2) Colmenares, J. C.; Luque, R. Heterogeneous Photocatalytic Nanomaterials: Prospects and Challenges in Selective Transformations of Biomass-derived Compounds. Chem. Soc. Rev. 2014, 43, 765778.

(3) Alivisatos, A. P. Semiconductor Clusters, Nanocrystals, and Quantum Dots. Science 1996, 271, 933-937.

(4) Michalet, X.; Pinaud, F. F.; Bentolila, L. A.; Tsay, J. M.; Doose, S.; Li, J. J.; Sundaresan, G.; Wu, A. M.; Gambhir, S. S.; Weiss, S. Quantum Dots for Live Cells, in Vivo Imaging, and Diagnostics. Science 2005, 307, 538-544.

(5) Feng, L.; Zhu, C.; Yuan, H.; Liu, L.; Lv, F.; Wang, S. Conjugated Polymer Nanoparticles: Preparation, Properties, Functionalization and Biological Applications. Chem. Soc. Rev. 2013, 42, 6620-6633.

(6) Hedley, G. J.; Ward, A. J.; Alekseev, A.; Howells, C. T.; Martins, E. R.; Serrano, L. A.; Cooke, G.; Ruseckas, A.; Samuel, I. D. W. Determining the Optimum Morphology in High-performance Polymer-Fullerene Organic Photovoltaic Cells. Nat. Commun. 2013, 4, 2867.

(7) Zhang, L. Z.; Djerdj, I.; Cao, M.; Antonietti, M.; Niederberger, M. Nonaqueous Sol-Gel Synthesis of a Nanocrystalline $\mathrm{InNbO}_{4}$ Visible-Light Photocatalyst. Adv. Mater. 2007, 19, 2083-2086.

(8) Miyauchi, M.; Nakajima, A.; Watanabe, T.; Hashimoto, K. Photocatalysis and Photoinduced Hydrophilicity of Various Metal Oxide Thin Films. Chem. Mater. 2002, 14, 2812-2816.
(9) Yao, J.; Yang, M.; Duan, Y. Chemistry, Biology, and Medicine of Fluorescent Nanomaterials and Related Systems: New Insights into Biosensing, Bioimaging, Genomics, Diagnostics, and Therapy. Chem. Rev. 2014, 114, 6130-6178.

(10) Jun, B.-H.; Hwang, D. W.; Jung, H. S.; Jang, J.; Kim, H.; Kang, H.; Kang, T.; Kyeong, S.; Lee, H.; Jeong, D. H.; Kang, K. W.; Youn, H.; Lee, D. S.; Lee, Y.-S. Ultrasensitive, Biocompatible, QuantumDot-Embedded Silica Nanoparticles for Bioimaging. Adv. Funct. Mater. 2012, 22, 1843-1849.

(11) Nuraje, N.; Dang, X.; Qi, J.; Allen, M. A.; Lei, Y.; Belcher, A. M. Biotemplated Synthesis of Perovskite Nanomaterials for Solar Energy Conversion. Adv. Mater. 2012, 24, 2885-2889.

(12) Ma, Y.; Wang, X.; Jia, Y.; Chen, X.; Han, H.; Li, C. Titanium Dioxide-Based Nanomaterials for Photocatalytic Fuel Generations. Chem. Rev. 2014, 114, 9987-10043.

(13) Zhou, Z.; Zhang, Y.; Shen, Y.; Liu, S.; Zhang, Y. Molecular Engineering of Polymeric Carbon Nitride: Advancing Applications from Photocatalysis to Biosensing and More. Chem. Soc. Rev. 2018, 47, 2298-2321.

(14) Wang, X.; Maeda, K.; Thomas, A.; Takanabe, K.; Xin, G.; Carlsson, J. M.; Domen, K.; Antonietti, M. A Metal-free Polymeric Photocatalyst for Hydrogen Production from Water under Visible Light. Nat. Mater. 2008, 8, 76-80.

(15) Ellis, J. E.; Sorescu, D. C.; Burkert, S. C.; White, D. L.; Star, A. Uncondensed Graphitic Carbon Nitride on Reduced Graphene Oxide for Oxygen Sensing via a Photoredox Mechanism. ACS Appl. Mater. Interfaces 2017, 9, 27142-27151.

(16) Hu, Y.; Shim, Y.; Oh, J.; Park, S.; Park, S.; Ishii, Y. Synthesis of 13C-,15N-Labeled Graphitic Carbon Nitrides and NMR-Based Evidence of Hydrogen-Bonding Assisted Two-Dimensional Assembly. Chem. Mater. 2017, 29, 5080-5089.

(17) Goettmann, F.; Thomas, A.; Antonietti, M. Metal-Free Activation of $\mathrm{CO}_{2}$ by Mesoporous Graphitic Carbon Nitride. Angew. Chem., Int. Ed. 2007, 46, 2717-2720.

(18) Kuriki, R.; Ishitani, O.; Maeda, K. Unique Solvent Effects on Visible-Light $\mathrm{CO}_{2}$ Reduction over Ruthenium(II)-Complex/Carbon Nitride Hybrid Photocatalysts. ACS Appl. Mater. Interfaces 2016, 8, 6011-6018.

(19) Sun, J.; Schmidt, B. V. K. J.; Wang, X.; Shalom, M. SelfStanding Carbon Nitride-Based Hydrogels with High Photocatalytic Activity. ACS Appl. Mater. Interfaces 2017, 9, 2029-2034.

(20) Yang, S.; Gong, Y.; Zhang, J.; Zhan, L.; Ma, L.; Fang, Z.; Vajtai, R.; Wang, X.; Ajayan, P. M. Exfoliated Graphitic Carbon Nitride Nanosheets as Efficient Catalysts for Hydrogen Evolution Under Visible Light. Adv. Mater. 2013, 25, 2452-2456.

(21) Son, E. J.; Lee, S. H.; Kuk, S. K.; Pesic, M.; Choi, D. S.; Ko, J. W.; Kim, K.; Hollmann, F.; Park, C. B. Carbon Nanotube-Graphitic Carbon Nitride Hybrid Films for Flavoenzyme-Catalyzed Photoelectrochemical Cells. Adv. Funct. Mater. 2017, 28, 1705232.

(22) Kurpil, B.; Kumru, B.; Heil, T.; Antonietti, M.; Savateev, A. Carbon Nitride Creates Thioamides in High Yields by the Photocatalytic Kindler Reaction. Green Chem. 2018, 20, 838-842.

(23) Kumru, B.; Shalom, M.; Antonietti, M.; Schmidt, B. V. K. J. Reinforced Hydrogels via Carbon Nitride Initiated Polymerization. Macromolecules 2017, 50, 1862-1869.

(24) Fu, Q.; Ruan, Q.; McKenzie, T. G.; Reyhani, A.; Tang, J.; Qiao, G. G. Development of a Robust PET-RAFT Polymerization Using Graphitic Carbon Nitride $\left(\mathrm{g}-\mathrm{C}_{3} \mathrm{~N}_{4}\right)$. Macromolecules 2017, 50, 75097516.

(25) Kumru, B.; Molinari, V.; Dünnebacke, R.; Blank, K. G.; Schmidt, B. V. K. J. Extremely Compressible Hydrogel via Incorporation of Modified Graphitic Carbon Nitride. Macromol. Rapid Commun. 2018, 40, 1800712.

(26) Oh, J.; Lee, J. M.; Yoo, Y.; Kim, J.; Hwang, S.-J.; Park, S. New Insight of the Photocatalytic Behaviors of Graphitic Carbon Nitrides for Hydrogen Evolution and their Associations with Grain Size, Porosity, and Photophysical Properties. Appl. Catal., B 2017, 218, 349-358. 
(27) Yan, W.; Yan, L.; Jing, C. Impact of Doped Metals on Ureaderived $\mathrm{g}_{-} \mathrm{C}_{3} \mathrm{~N}_{4}$ for Photocatalytic Degradation of Antibiotics: Structure, Photoactivity and Degradation Mechanisms. Appl. Catal., B 2019, 244, 475-485.

(28) Qian, H.; Huang, H.; Wang, X. Design and Synthesis of Palladium/Graphitic Carbon Nitride/Carbon Black Hybrids as Highperformance Catalysts for Formic Acid and Methanol Electrooxidation. J. Power Sources 2015, 275, 734-741.

(29) Bu, X.; Li, J.; Yang, S.; Sun, J.; Deng, Y.; Yang, Y.; Wang, G.; Peng, Z.; He, P.; Wang, X.; Ding, G.; Yang, J.; Xie, X. Surface Modification of $\mathrm{C}_{3} \mathrm{~N}_{4}$ through Oxygen-Plasma Treatment: A Simple Way toward Excellent Hydrophilicity. ACS Appl. Mater. Interfaces 2016, 8, 31419-31425.

(30) Sun, J.; Phatake, R.; Azoulay, A.; Peng, G.; Han, C.; Barrio, J.; Xu, J.; Wang, X.; Shalom, M. Covalent Functionalization of Carbon Nitride Frameworks through Cross-Coupling Reactions. Chem.-Eur. J. 2018, 24, 14921-14927.

(31) Kim, J. K.; Park, S.; Yoo, R. J.; Jeong, H. J.; Oh, J.; Lee, Y. J.; Park, S.; Kim, D. W. Thin PEGylated Carbon Nitrides: WaterDispersible Organic Nanodots as Bioimaging Probes. Chem.-Eur. J. 2018, 24, 3506-3511.

(32) Zhang, Y.; Thomas, A.; Antonietti, M.; Wang, X. Activation of Carbon Nitride Solids by Protonation: Morphology Changes, Enhanced Ionic Conductivity, and Photoconduction Experiments. J. Am. Chem. Soc. 2009, 131, 50-51.

(33) Kumru, B.; Antonietti, M.; Schmidt, B. V. K. J. Enhanced Dispersibility of Graphitic Carbon Nitride Particles in Aqueous and Organic Media via a One-Pot Grafting Approach. Langmuir 2017, 33, 9897-9906.

(34) Kumru, B.; Cruz, D.; Heil, T.; Schmidt, B. V. K. J.; Antonietti, M. Electrostatic Stabilization of Carbon Nitride Colloids in Organic Solvents Enables Stable Dispersions and Transparent Homogeneous CN Films for Optoelectronics. J. Am. Chem. Soc. 2018, 140, 1753217537.

(35) Kumru, B.; Molinari, V.; Shalom, M.; Antonietti, M.; Schmidt, B. V. K. J. Tough High Modulus Hydrogels Derived from CarbonNitride via an Ethylene Glycol Co-solvent Route. Soft Matter 2018, 14, 2655-2664.

(36) Khire, V. S.; Yi, Y.; Clark, N. A.; Bowman, C. N. Formation and Surface Modification of Nanopatterned Thiol-ene Substrates using Step and Flash Imprint Lithography. Adv. Mater. 2008, 20, 33083313

(37) Delaittre, G.; Goldmann, A. S.; Mueller, J. O.; Barner-Kowollik, C. Efficient Photochemical Approaches for Spatially Resolved Surface Functionalization. Angew. Chem., Int. Ed. 2015, 54, 11388-11403.

(38) Narupai, B.; Page, Z. A.; Treat, N. J.; McGrath, A. J.; Pester, C. W.; Discekici, E. H.; Dolinski, N. D.; Meyers, G. F.; Read de Alaniz, J.; Hawker, C. J. Simultaneous Preparation of Multiple Polymer Brushes under Ambient Conditions using Microliter Volumes. Angew. Chem., Int. Ed. 2018, 57, 13433-13438.

(39) Abt, D.; Schmidt, B. V. K. J.; Pop-Georgievski, O.; Quick, A. S.; Danilov, D.; Kostina, N. Y.; Bruns, M.; Wenzel, W.; Wegener, M.; Rodriguez-Emmenegger, C.; Barner-Kowollik, C. Designing Molecular Printboards: A Photolithographic Platform for Recodable Surfaces. Chem.-Eur. J. 2015, 21, 13186-13190.

(40) Arumugam, S.; Orski, S. V.; Locklin, J.; Popik, V. V. Photoreactive Polymer Brushes for High-Density Patterned Surface Derivatization Using a Diels-Alder Photoclick Reaction. J. Am. Chem. Soc. 2011, 134, 179-182.

(41) Rodriguez-Emmenegger, C.; Preuss, C. M.; Yameen, B.; PopGeorgievski, O.; Bachmann, M.; Mueller, J. O.; Bruns, M.; Goldmann, A. S.; Bastmeyer, M.; Barner-Kowollik, C. Controlled Cell Adhesion on Poly(dopamine) Interfaces Photopatterned with Non-Fouling Brushes. Adv. Mater. 2013, 25, 6123-6127.

(42) Park, S.; Yousaf, M. N. An Interfacial Oxime Reaction To Immobilize Ligands and Cells in Patterns and Gradients to Photoactive Surfaces. Langmuir 2008, 24, 6201-6207.

(43) Pauloehrl, T.; Delaittre, G.; Winkler, V.; Welle, A.; Bruns, M.; Börner, H. G.; Greiner, A. M.; Bastmeyer, M.; Barner-Kowollik, C.
Adding Spatial Control to Click Chemistry: Phototriggered DielsAlder Surface (Bio)functionalization at Ambient Temperature. Angew. Chem., Int. Ed. 2011, 51, 1071-1074.

(44) Page, Z. A.; Narupai, B.; Pester, C. W.; Bou Zerdan, R.; Sokolov, A.; Laitar, D. S.; Mukhopadhyay, S.; Sprague, S.; McGrath, A. J.; Kramer, J. W.; Trefonas, P.; Hawker, C. J. Novel Strategy for Photopatterning Emissive Polymer Brushes for Organic Light Emitting Diode Applications. ACS Cent. Sci. 2017, 3, 654-661.

(45) Cao, S.; Low, J.; Yu, J.; Jaroniec, M. Polymeric Photocatalysts Based on Graphitic Carbon Nitride. Adv. Mater. 2015, 27, 21502176.

(46) Kreider, K. G.; Tarlov, M. J.; Gillen, G. J.; Poirier, G. E.; Robins, L. H.; Ives, L. K.; Bowers, W. D.; Marinenko, R. B.; Smith, D. T. Sputtered Amorphous Carbon Nitride Films. J. Mater. Res. 1995, 10, 3079-3083.

(47) Arazoe, H.; Miyajima, D.; Akaike, K.; Araoka, F.; Sato, E.; Hikima, T.; Kawamoto, M.; Aida, T. An Autonomous Actuator Driven by Fluctuations in Ambient Humidity. Nat. Mater. 2016, 15, 1084.

(48) Bian, J.; Li, Q.; Huang, C.; Li, J.; Guo, Y.; Zaw, M.; Zhang, R.Q. Thermal Vapor Condensation of Uniform Graphitic Carbon Nitride Films with Remarkable Photocurrent Density for Photoelectrochemical Applications. Nano Energy 2015, 15, 353-361.

(49) Zhang, J.; Zhang, M.; Lin, L.; Wang, X. Sol Processing of Conjugated Carbon Nitride Powders for Thin-Film Fabrication. Angew. Chem., Int. Ed. 2015, 54, 6297-6301.

(50) Shalom, M.; Gimenez, S.; Schipper, F.; Herraiz-Cardona, I.; Bisquert, J.; Antonietti, M. Controlled Carbon Nitride Growth on Surfaces for Hydrogen Evolution Electrodes. Angew. Chem. 2014, 126, 3728-3732.

(51) Peng, G.; Xing, L.; Barrio, J.; Volokh, M.; Shalom, M. A General Synthesis of Porous Carbon Nitride Films with Tunable Surface Area and Photophysical Properties. Angew. Chem., Int. Ed. 2018, 57, 1186-1192.

(52) Barrio, J.; Shalom, M. Rational Design of Carbon Nitride Materials by Supramolecular Preorganization of Monomers. ChemCatChem 2018, 10, 5573-5586.

(53) Fang, Y.; Li, X.; Wang, X. Synthesis of Polymeric Carbon Nitride Films with Adhesive Interfaces for Solar Water Splitting Devices. ACS Catal. 2018, 8, 8774-8780.

(54) Jia, L.; Wang, H.; Dhawale, D.; Anand, C.; Wahab, M. A.; Ji, Q.; Ariga, K.; Vinu, A. Highly ordered macro-mesoporous carbon nitride film for selective detection of acidic/basic molecules. Chem. Commun. 2014, 50, 5976-5979.

(55) Zheng, D.; Cao, X.-N.; Wang, X. Precise Formation of a Hollow Carbon Nitride Structure with a Janus Surface To Promote Water Splitting by Photoredox Catalysis. Angew. Chem., Int. Ed. 2016, 55, $11512-11516$

(56) Zheng, D.; Pang, C.; Liu, Y.; Wang, X. Shell-engineering of Hollow $\mathrm{g}-\mathrm{C}_{3} \mathrm{~N}_{4}$ Nanospheres via Copolymerization for Photocatalytic Hydrogen Evolution. Chem. Commun. 2015, 51, 9706-9709.

(57) Liu, J.; Wang, H.; Chen, Z. P.; Moehwald, H.; Fiechter, S.; van de Krol, R.; Wen, L.; Jiang, L.; Antonietti, M. Microcontact-PrintingAssisted Access of Graphitic Carbon Nitride Films with Favorable Textures toward Photoelectrochemical Application. Adv. Mater. 2014, 27, 712-718.

(58) Martin-Gallego, M.; Verdejo, R.; Gestos, A.; Lopez-Manchado, M. A.; Guo, Q. Morphology and Mechanical Properties of Nanostructured Thermoset/Block Copolymer Blends with Carbon Nanoparticles. Composites, Part A 2015, 71, 136-143.

(59) Sadler, J. M.; Toulan, F. R.; Palmese, G. R.; La Scala, J. J. Unsaturated Polyester Resins for Thermoset Applications Using Renewable Isosorbide as a Component for Property Improvement. J. Appl. Polym. Sci. 2015, 132, 42315.

(60) Hazarika, A.; Deka, B. K.; Kim, D.; Roh, H. D.; Park, Y.-B.; Park, H. W. Fabrication and Synthesis of Highly Ordered Nickel Cobalt Sulfide Nanowire-Grown Woven Kevlar Fiber/Reduced Graphene Oxide/Polyester Composites. ACS Appl. Mater. Interfaces 2017, 9, 36311-36319. 
(61) Chu, F.; Zhang, D.; Hou, Y.; Qiu, S.; Wang, J.; Hu, W.; Song, L. Construction of Hierarchical Natural Fabric Surface Structure Based on Two-Dimensional Boron Nitride Nanosheets and Its Application for Preparing Biobased Toughened Unsaturated Polyester Resin Composites. ACS Appl. Mater. Interfaces 2018, 10, 40168-40179.

(62) Shalom, M.; Inal, S.; Fettkenhauer, C.; Neher, D.; Antonietti, M. Improving Carbon Nitride Photocatalysis by Supramolecular Preorganization of Monomers. J. Am. Chem. Soc. 2013, 135, 71187121.

(63) Barrio, J.; Lin, L.; Amo-Ochoa, P.; Tzadikov, J.; Peng, G.; Sun, J.; Zamora, F.; Wang, X.; Shalom, M. Unprecedented CentimeterLong Carbon Nitride Needles: Synthesis, Characterization and Applications. Small 2018, 14, 1800633.

(64) Peng, G.; Volokh, M.; Tzadikov, J.; Sun, J.; Shalom, M. Carbon Nitride/Reduced Graphene Oxide Film with Enhanced Electron Diffusion Length: An Efficient Photo-Electrochemical Cell for Hydrogen Generation. Adv. Energy Mater. 2018, 8, 1800566.

(65) Song, L.; Zhang, S.; Wu, X.; Tian, H.; Wei, Q. Graphitic $\mathrm{C}_{3} \mathrm{~N}_{4}$ Photocatalyst for Esterification of Benzaldehyde and Alcohol under Visible Light Radiation. Ind. Eng. Chem. Res. 2012, 51, 9510-9514.

(66) Xu, J.; Chen, T.; Wang, X.; Xue, B.; Li, Y.-X. Preparation of mesoporous graphitic carbon nitride using hexamethylenetetramine as a new precursor and catalytic application in the transesterification of $\beta$-keto esters. Catal. Sci. Technol. 2014, 4, 2126-2133.

(67) Jung, J. H.; Ree, M.; Kim, H. Acid- and Base-Catalyzed Hydrolyses of Aliphatic Polycarbonates and Polyesters. Catal. Today 2006, 115, 283-287.

(68) Volokh, M.; Peng, G.; Barrio, J.; Shalom, M. Carbon Nitride Materials for Water Splitting Photoelectrochemical Cells. Angew. Chem., Int. Ed. 2018, DOI: 10.1002/anie.201806514.

(69) Safaei, J.; Mohamed, N. A.; Mohamad Noh, M. F.; Soh, M. F.; Ludin, N. A.; Ibrahim, M. A.; Roslam Wan Isahak, W. N.; Mat Teridi, M. A. Graphitic Carbon Nitride $\left(\mathrm{g}-\mathrm{C}_{3} \mathrm{~N}_{4}\right)$ Electrodes for Energy Conversion and Storage: A Review on Photoelectrochemical Water Splitting, Solar Cells and Supercapacitors. J. Mater. Chem. A 2018, 6, 22346-22380.

(70) Fang, Y.; Xu, Y.; Li, X.; Ma, Y.; Wang, X. Coating Polymeric Carbon Nitride Photoanodes on Conductive Y:ZnO Nanorod Arrays for Overall Water Splitting. Angew. Chem., Int. Ed. 2018, 57, 97499753.

(71) Xiong, W.; Chen, S.; Huang, M.; Wang, Z.; Lu, Z.; Zhang, R.Q. Crystal-Face Tailored Graphitic Carbon Nitride Films for HighPerformance Photoelectrochemical Cells. ChemSusChem 2018, 11, 2497-2501. 